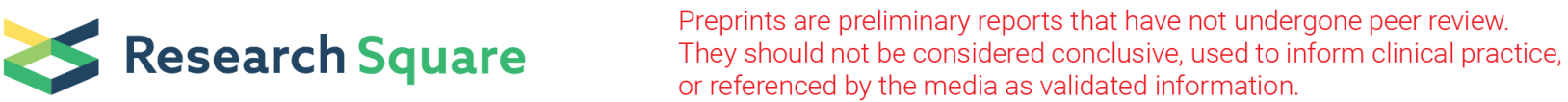 \\ Unified energy law for fluctuating density wave orders in cuprate pseudogap phase
}

\section{Rong Li}

Peking University

Zhen-Su She ( $\nabla$ she@pku.edu.cn )

Peking University https://orcid.org/0000-0001-7001-9995

\section{Article}

Keywords: pseudogap, density wave order, unified energy law

Posted Date: August 9th, 2021

DOI: https://doi.org/10.21203/rs.3.rs-725614/v1

License: (c) (1) This work is licensed under a Creative Commons Attribution 4.0 International License. Read Full License

Version of Record: A version of this preprint was published at Communications Physics on January 11th, 2022. See the published version at https://doi.org/10.1038/s42005-021-00789-9. 


\title{
Unified energy law for fluctuating density wave orders in cuprate pseudogap phase
}

\author{
Rong $\mathrm{Li}$ and Zhen-Su She* \\ State Key Laboratory for Turbulence and Complex Systems, \\ College of Engineering, Peking University, Beijing 100871, China.
}

\begin{abstract}
The origin of the pseudogap and its relationship to symmetry-broken orders in cuprates have been extensively debated. Here, we report a unified energy law underlying the pseudogap, which determines the scattering rate, pseudogap energy, and its onset temperature, with a quadratic scaling of the wavevector of density wave order (DWO). The law is validated by data from over one hundred samples, and a further prediction that the master order of pseudogap transforms from fluctuating spin to charge DWO is also confirmed by independent measurements. Furthermore, the energy law enables our derivation of the well-known linear scalings for the resistivity of the strange metal phase and the transition temperature of the superconducting phase, shedding light on the universal origin of various phases. Finally, it is concluded that fluctuating orders provide a critical bridge linking microscopic spectra to macroscopic transport in cuprates, showing promise for the quantification of other strongly correlated materials.
\end{abstract}

\section{Introduction}

A central puzzle of high-temperature cuprate superconductors is the pseudogap $\Delta^{*}$ that occurs below a phase transition at $T^{*}$, characterized by a suppression of the electronic density of states around the Fermi level $[1,2]$. The critical question that remains unanswered is, what is the underlying quantum order that determines the pseudogap? Experimentally, in the pseudogap phase, two distinct classes of symmetry-breaking orders are widely reported $[1,3-5]$, namely, zero wavevector $Q=0$ states (e.g., nematicity and loop current) and the finite wavevector $Q \neq 0$ density wave order (DWO). From a mean-field viewpoint, the DWO breaks translational symmetry to produce an anisotropic gap, and small Fermi surface (FS) pockets $[1,6,7]$. However, experimentally observed static (charge and spin) DWOs exhibit an onset temperature significantly lower than $T^{*}$ and no further gap opening [1,2], apparently ruling out the static DWO as the origin of the pseudogap [3, 8]. On the other hand, the nematic and loop current orders are widely observed to emerge coincidentally during the pseudogap opening at $T^{*}[4,5]$. However, within the mean-field theory, these intra-unit-cell orders are known to be unable to break the lattice translation symmetry to open a pseudogap $[9,10]$. Thus, the controversy of whether the pseudogap opening can be related to the conventional form of these two classes [1] remains to be solved.

Recent theoretical advances suggest that the pseudogap phase can be understood with intertwined orders, including the $Q \neq 0$ DWO and the $Q=0$ nematic, loop-current or superconducting states $[8,9,11-$ 13]. For instance, it was demonstrated that a partially melted unidirectional DWO (either spin or charge) could generate a vestigial nematic phase [13]. In this context, using elaborate data analysis, various experiments were performed to achieve precise measurements of energy scales and wavevectors associated with a charge density wave (CDW), revealing an intimate link among CDWs, the nematic order, and the pseudogap. Specifically, recent Raman measurements of the spectral gap associated with CDWs $\left(\Delta_{\mathrm{CDW}}\right)$ in $\mathrm{Bi}_{2} \mathrm{Sr}_{2} \mathrm{CaCu}_{2} \mathrm{O}_{8+\delta}(\mathrm{Bi}-2212), \mathrm{HgBa}_{2} \mathrm{CuO}_{4+\delta}(\mathrm{Hg}-2201)$, $\mathrm{HgBa}_{2} \mathrm{Ca}_{2} \mathrm{Cu}_{3} \mathrm{O}_{8+\delta}(\mathrm{Hg}-1223)$ and $\mathrm{YBa}_{2} \mathrm{Cu}_{3} \mathrm{O}_{6+\delta}(\mathrm{Y}-$ $123)$, display the same doping dependence as the pseudogap energy $[14,15]$, indicating that the pseudogap and the CDW energy scale may have a common microscopic origin. Furthermore, by analysing tunnelling conductance from distinct regions of momentum space, Mukhopadhyay et al. identified energies characterizing the CDW, nematicity, and pseudogap in Bi-2212 and found that they are identical [16], which suggested that the pseudogap may originate from highly disordered unidirectional DWO. This viewpoint was further supported by more recent observations from scanning tunnelling microscopy (STM) and nuclear magnetic resonance (NMR): the CDW phase is locally unidirectional (with significant phase fluctuations globally) for underdoped Bi-2212 [17-19] and Y-123 [20], and the local spectral gap in $(\mathrm{Bi}, \mathrm{Pb})_{2}(\mathrm{Sr}, \mathrm{La})_{2} \mathrm{CuO}_{6+\delta}(\mathrm{Pb}-\mathrm{Bi} 2201)$ is positively correlated with fluctuating $\mathrm{CDW}$ wavevector [21].

While the abovementioned theoretical and experimental progress indicates a mainly qualitative link between the CDW and the pseudogap and highlights the crucial role of phase fluctuations (either thermal fluctuations or spatial disorders) in this link, we are devoted to investigating whether there exists a universal quantitative relationship between energy scales of the pseudogap and the fluctuating DWO. In this context, three critical challenges remain to be addressed $[15,22]$. First, one needs to define the energy scales linked to the intrinsic nature of DWO to determine whether the measured gap is from the CDW rather than other orders. Second, one needs to have a clear explanation for the origin of the universal doping dependence of $\Delta^{*}$ and $T^{*}$. Finally, one needs to explain the transition from a CDW to a spin density wave (SDW) and its influence on the pseudo- 
gap $[1,23,24]$. To answer these questions, we naturally introduce the wavevector and amplitude as the two fundamental quantities to characterize DWO and to investigate their relationships with two critical energy scales in the single-particle self-energy (see section 1 in the "Methods"), namely, the gap energy and the scattering rate.

To derive these relationships, we use an innovative symmetry-breaking analysis inspired by a recent successful wall turbulence theory [25]. Similar to eddies exposed to strongly wall-constrained shear turbulence, the fluctuating DWO near $T^{*}$ has a complex spatiotemporal pattern, which is generally difficult to model by an order parameter. However, the structural ensemble dynamics (SED) theory of wall turbulence [25, 26] states that eddy motions can be effectively self-organized into several statistical ensembles. This self-organization comes from the constraint of symmetry (e.g., dilation associated with wall turbulence) and is expressed by the fact that eddy lengths satisfy generalized power laws, which determine the crucial energy distribution [26] and transport coefficient [25]. Therefore, we assume that the pseudogap energy scales should satisfy a power-law relation with the wavevector (or wavelength) of the fluctuating DWO. Specifically, as ubiquitously observed in cuprates [3, 8, 23, 27], the mesoscopic (fluctuating) DWO emerges as a consequence of translational symmetry-breaking. Its wavelength $l_{\mathrm{o}}=2 \pi / Q_{\mathrm{o}}$ corresponds to a small wavevector $Q_{\mathrm{o}}$ (smaller than the reciprocal lattice vector, where o represents the order type), which connects momentum states on the FS, favouring the Umklapp scattering associated with $Q_{\mathrm{o}}$ to determine the pseudogap energy scales in the single-particle selfenergy.

In this article, we first introduce an Umklapp scattering mechanism to derive a quadratic scaling with the DWO wavevector for the scattering rate and further extend it to the pseudogap energy $\Delta^{*}$ and the onset temperature $T^{*}$. The scalings are then validated by the spectral gap and the onset temperature data obtained for over one hundred samples (see section 3 in the "Methods"), indicating that the universal monotonic decrease with increasing doping of $\Delta^{*}$ and $T^{*}$ in the intermediate doping regime originates from the variation in the CDW wavevector $Q_{\mathrm{CDW}}$. Furthermore, by using resistivity data of high-quality single crystals, we demonstrate the validity of the quadratic scaling, with a universal scattering coefficient for both the CDW and SDW. Finally, by using the energy law, we can derive a length, from the resistivity and pseudogap energy data (with the so-called length mapping method), of the master order, which displays a universal transition in the pseudogap phase from the SDW (at light doping) to the CDW (at intermediate doping), as confirmed by independent measurements.

Furthermore, the new energy law offers a straightfor-

ward explanation for both the strange metal resistivity and the linear scaling between the superconducting transition temperature and superfluid density, indicating that the law is universal for all three phases of hole-doped cuprates. These findings let us conclude that mesoscopic (fluctuating) orders provide a crucial bridge (in universal energy scaling) linking microscopic spectra to macroscopic transport in cuprates. This discovery offers a potential breakthrough towards a comprehensive theoretical description of strongly correlated materials.

\section{Results}

Universal energy law for fluctuating DWO. The fluctuating DWO generates phason modes to induce carrier scattering, which requires conservation of momentum as $\mathbf{k}^{\prime}=\mathbf{k}+\mathbf{q}+n \mathbf{Q}_{\mathrm{o}}$, where $\mathbf{k}$ and $\mathbf{k}^{\prime}$ are the initial and final states, $\mathbf{q}$ is the phason wavevector, and $n$ is a positive integer. Ignoring the anisotropy of the scattering rate $\Gamma_{\mathbf{k}}$, we consider only the mean scattering rate $\Gamma$, which is independent of $\mathbf{k}, \mathbf{k}^{\prime}$, and $\mathbf{q}$ but closely related to $Q_{\mathrm{o}}$ in an Umklapp scattering process. This yields an energy law for the mean scattering rate as follows:

$$
\Gamma=\gamma_{\Gamma} \frac{\hbar^{2} Q_{\mathrm{o}}^{2}}{m^{*}}=\gamma_{\Gamma} \frac{h^{2}}{m^{*} l_{\mathrm{o}}^{2}},
$$

where $\gamma_{\Gamma}$ is a dimensionless coefficient describing the mean scattering strength and $m^{*}$ is the effective mass of a carrier.

Eq. (1) is not only the most straightforward explicit function of $\Gamma\left(Q_{\mathrm{o}}\right)$ with correct dimensionality and inversion symmetry but also a natural result of the Umklapp scattering theory under the small momentum difference and large sound velocity approximation (see section 2 in the "Methods"). The scattering theory also predicts that $\gamma_{\Gamma}$ is proportional to the module square of the carrier-DWO coupling. Based on the $t-J$ model [28], we assume that this coupling is proportional to the ratio between the superexchange energy $J$ and the hopping energy $t$, which yields:

$$
\gamma_{\Gamma}=A_{\Gamma} \frac{J^{2}}{t^{2}} \approx 0.11 A_{\Gamma},
$$

where the dimensionless coefficient $A_{\Gamma}$ is proportional to the phason amplitude. The second equality originates from the approximation $J / t \approx 1 / 3$, supported by ab initio calculations and Raman scattering data $[29,30]$. Generally, $A_{\Gamma}$ depends on temperature and doping. However, these contributions may be renormalized in the intermediate temperature and doping regime discussed below (see also the Discussion section). Therefore, $\gamma_{\Gamma}$ and the energy law may be approximately universal.

Furthermore, as for the scattering rate, we propose that the characteristic gap amplitude $\Delta$ originates from 
the Umklapp scattering by the fluctuating DWO. Therefore, $\Delta$ can be predicted from Eq. (1) by extending the scattering source from order fluctuations to its mean field, which involves only the substitution of the amplitude of phason modes at $q \neq 0$ in Eq. (2) for the amplitude of modes at $q=0$ without further calculations. Thus, we obtain an energy law similar to Eq. (1):

$$
\Delta=\gamma_{\Delta} \frac{\hbar^{2} Q_{\mathrm{o}}^{2}}{m^{*}}=\gamma_{\Delta} \frac{h^{2}}{m^{*} l_{\mathrm{o}}^{2}},
$$

Here, $\gamma_{\Delta}=J^{2} / t^{2} A_{\Delta} \approx 0.11 A_{\Delta}$ is the dimensionless coefficient describing the scattering strength, where $A_{\Delta}$ is a dimensionless parameter proportional to the amplitude of the mean-field.

From a pairing perspective, the DWO is equivalent to pairing in the particle-hole channel [31]. In this context, we define the onset temperature $T^{*}$ of the pseudogap opening with the emergence of this particle-hole pairing. This implies that the thermal-fluctuation energy $k_{\mathrm{B}} T^{*}$ is linearly proportional to the pseudogap energy, as widely observed in spectroscopic measurements $[15,32]$. This naturally yields a relationship for determining $T^{*}$, as follows:

$$
T^{*}=\gamma_{\mathrm{T}} \frac{\hbar^{2} Q_{\mathrm{o}}^{2}}{m^{*}}=\gamma_{\mathrm{T}} \frac{h^{2}}{m^{*} l_{\mathrm{o}}^{2}},
$$

where $\gamma_{\mathrm{T}} \propto \gamma_{\Delta}$ is a dimensionless coefficient. In ${ }^{51}$ addition, in the following, we express $Q_{\mathrm{o}}$ in units of ${ }^{52}$ $2 \pi / a_{0}$, where $a_{0}$ is the in-plane lattice constant.

Gap energy scales associated with the CDW. The CDW has been identified as a leading competitor of superconductivity in cuprates [3]. Therefore, it would be intriguing to examine Eq. (3) and Eq. (4) to evaluate whether the CDW is indeed related to the pseudogap and satisfies these simple energy laws. Recently, using STM, Webb et al. carried out simultaneous measurements of the spectral gap and $Q_{\mathrm{CDW}}$ for local charge modulations in $(\mathrm{Bi}, \mathrm{Pb})_{2}(\mathrm{Sr}, \mathrm{La})_{2} \mathrm{CuO}_{6+\delta}(\mathrm{Pb}-\mathrm{Bi} 2201)$ at a superconducting state (at $6 \mathrm{~K}$ ) [21], providing an appropriate sample for this evaluation. $m^{*}=2.7 m_{\mathrm{e}}$ for Bi-2201 based on the optical conductivity measurement in Ref. [33] and $\gamma_{\Delta}=0.135$, Eq. (3) were used to predict a quadratic scaling, $\Delta=\hbar^{2} Q_{\mathrm{CDW}}^{2} / 20 m_{\mathrm{e}}$, that is quantitatively consistent with the majority of the reported data shown in Fig. 1. This agreement confirms the quadratic scaling between $\Delta$ and $Q_{\mathrm{CDW}}$.

It is interesting to compare the present prediction with previous theories. In the past, the connection between the pseudogap and $Q_{\mathrm{CDW}}$ was attributed to fermiology, which explains the decrease in $Q_{\mathrm{CDW}}$ with increasing doping as a result of a growing hole pocket $[34,35]$. The predictions of two models based on fermiology [21] are presented in Fig. 1. In the antinodal $(\mathrm{AN})$ case, $Q_{\mathrm{CDW}}$ connects the nested antinodal segments of the FS, and in the antiferromagnetic zone boundary (AFZB) case,

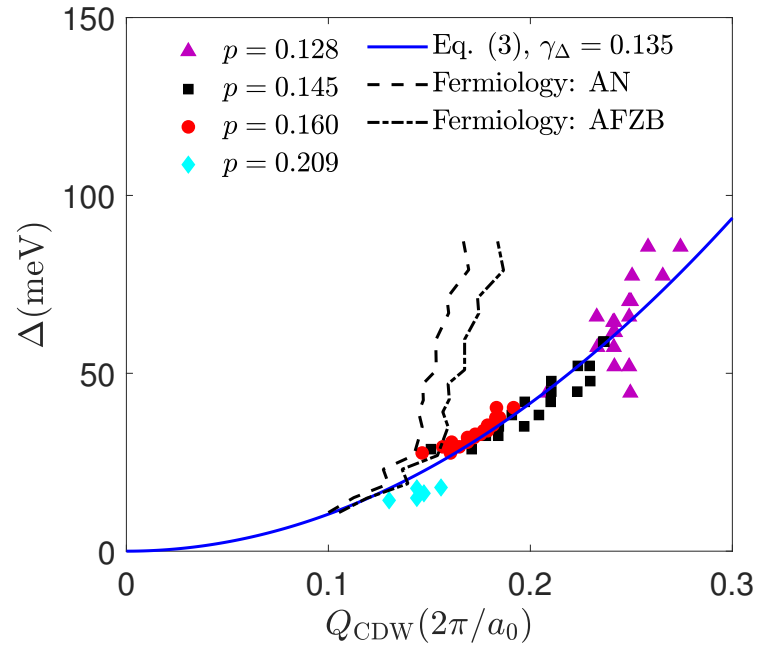

FIG. 1. Scaling between the pseudogap and the CDW vector. The symbols represent the $\mathrm{Pb}-\mathrm{Bi} 2201$ data determined from STM measurements in Ref. [21]. The solid blue line represents the prediction from Eq. (3) with the fitting parameter $\gamma_{\Delta}=0.135$ and $m^{*}=2.7 m_{\mathrm{e}}$. The dashed and dash-dotted lines extracted from Ref. [21] represent the Fermiology-driven wavevectors.

$Q_{\mathrm{CDW}}$ connects the points at which the FS crosses the AFZB. Both models show apparent overestimation for the optimal and underdoped regimes. Therefore, the present proposal that the spectral gap associated with CDW originates from scattering by CDW rather than the simple FS instability is well confirmed. In addition, one may wonder whether the global incommensurability is compatible with recent STM and NMR observations that the CDW is locally commensurate for underdoped Bi-2212 and Y-123 [17-20]. Assuming phase fluctuations to be of Gaussian type, we derive from Eq. (3) a gap distribution that is quite similar to the experimental observations (see Supplementary Note 2), implying that the present energy law applies to the locally commensurate CDW and the accompanying phase fluctuations.

Note that local STM measurement of the superconducting state includes three effects, namely, the CDW gap, the pseudogap, and the superconducting gap. Thus, it is important to find a way to distinguish these gaps. The recent Raman response measurements [14, 15] defined the CDW gap $\left(\Delta_{\mathrm{CDW}}\right)$ as the nodal hump location above the superconducting transition temperature $T_{\mathrm{c}}$, the high-energy scale pseudogap $\Delta_{\mathrm{H}}^{*}$ as the antinodal depletion location above $T_{\mathrm{c}}$, and the antinodal superconducting gap $\Delta_{\mathrm{SC}}^{\mathrm{AN}}$ as the antinodal peak location below $T_{\mathrm{c}}$, as shown in Fig. 2. Interestingly, with simple normalizations (i.e., divide energy by constant), the experimental data for all three gaps in $\mathrm{Hg}-2201$ (hollow symbols) and Bi-2212 (solid symbols) collapse to the prediction of Eq. (3) (solid blue line). The fitting 


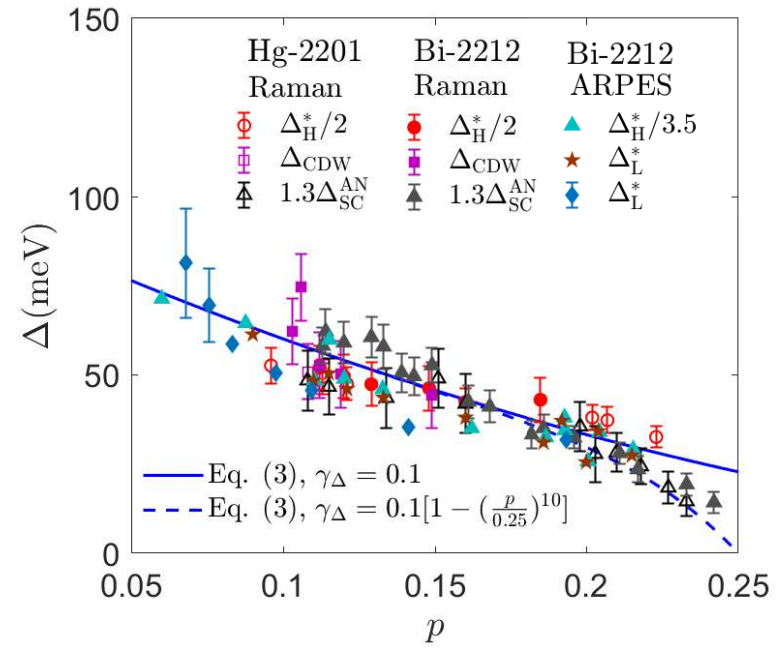

FIG. 2. Various gap energy scales. The symbols represent the low- and high-energy scales of the pseudogap, the CDW gap and the antinodal superconducting gap of Hg-2201 (hollow symbols) and Bi-2212 (solid symbols) determined from the Raman response in Ref. [15] and the ARPES data in Ref. [32, 39]. The solid blue line represents the prediction from Eq. (3) with the fitting parameter $\gamma_{\Delta}=0.1$ and $m^{*}=2.45 m_{\mathrm{e}}$, a linear model $Q_{\mathrm{CDW}}=0.343-0.699 p$ and the in-plane lattice constant $a_{0}=3.89 \AA$ for $\mathrm{Hg}-2201$ [40]. The dashed blue line includes a quick decay of the CDW amplitude near the pseudogap QCP. parameters are $\gamma_{\Delta}=0.1, m^{*}=2.45 m_{\mathrm{e}}$ (determined from the quantum oscillation experiment of $\mathrm{Hg}-2201$ at $\mathrm{p}=0.09$ [36]) and $Q_{\mathrm{CDW}}=0.343-0.699 p$ (linearly fitted from the experimental data for Hg-2201 in Refs. [37, 38]; see Supplementary Note 1). On the other hand, the angle resolved photoemission spectroscopy (ARPES) measurement in the antinodal direction usually suggests two energy scales for the pseudogap, namely, the low-energy scale $\left(\Delta_{\mathrm{L}}^{*}\right.$, e.g., the peak location) and the high-energy scale $\left(\Delta_{\mathrm{H}}^{*}\right.$, e.g., the hump location) [39]. It is remarkable that both energy scales for Bi-2212 determined from Ref. $[32,39]$ follow the doping dependence of our prediction as well.

Fig. 1 and Fig. 2 contain three types (STM, Raman, and ARPES) of data for over fifty samples of three compounds; thus, our theory is firmly validated. More importantly, we uncover the relationship that the spectral gaps associated with the pseudogap and the antinodal superconductivity have a universal quadratic scaling with the CDW wavevector, i.e., $\Delta_{\mathrm{H}}^{*} \propto \Delta_{\mathrm{L}}^{*} \approx$ $\Delta_{\mathrm{CDW}} \propto Q_{\mathrm{CDW}}^{2}$ and $\Delta_{\mathrm{SC}}^{\mathrm{AN}} \sim \Delta_{\mathrm{CDW}} \propto Q_{\mathrm{CDW}}^{2}$. This finding reveals that the pseudogap and antinodal superconductivity in the intermediate doping regime are both governed by the scattering by the CDW. In addition, since the pseudogap onset temperature is the gap opening temperature, $T^{*}$ should also satisfy the same quadratic scaling. As shown in Fig. 3, the predictions
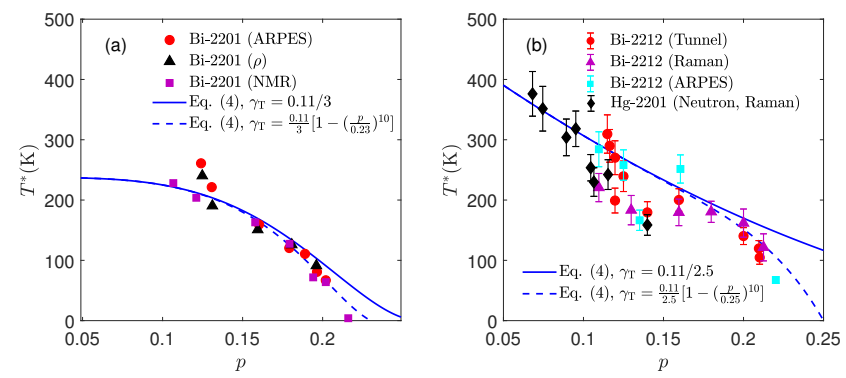

FIG. 3. Doping dependence of the pseudogap onset temperature $T^{*}$. (a) The symbols represent Bi-2201 data determined from Ref. [41]. The solid blue line represents the predictions from Eq. (4) with $\gamma_{\mathrm{T}}=0.11 / 3$, the same $m^{*}$ used in Fig. 1 , and a power-law model $Q_{\mathrm{CDW}}=0.269\left[1-(p / 0.261)^{3.79}\right]$. (b) The symbols represent Bi-2212 and Hg-2201 data determined from Ref. [15]. The solid blue line represents the prediction from Eq. (4) with $\gamma_{\mathrm{T}}=0.11 / 2.5$ and the same $m^{*}$ and $Q_{\mathrm{CDW}}$ used in Fig. 2. The dashed blue lines indicate the quick decay of the CDW amplitude near the pseudogap QCP.

28 from Eq. (4) are indeed consistent with experimental 29 data over a wide doping range of Bi-2201, Bi-2212 and Hg-2201 [15, 41], confirming the universality of the energy law. Note that in the fitting of Bi-2201, a power-law model $Q_{\mathrm{CDW}}=0.269\left[1-(p / 0.261)^{3.79}\right]$ fitted from the experimental data in Refs. [24, 42] has been used (see Supplementary Note 1).

Our findings reveal that, in the CDW-dominated regime, the well-known monotonic decreases in $\Delta$ and $T^{*}$ for three compounds with short-range DWOs mainly result from the reduction of the CDW wavevector with increasing doping. Note also that the experimental data begin to deviate from the prediction at $p \sim 0.2$, likely because the CDW amplitude begins to decrease noticeably near the pseudogap quantum critical point (QCP), which might be described by the defect power law of $\gamma_{\Delta}$ (the dashed blue lines in Figs. 2 and 3). Furthermore, near $p \sim 1 / 8, T^{*}$ for Bi-2212 and Hg-2201 is lower than our prediction, which may reveal an anomaly in the CDW gap opening. Its relevance to the temperature and doping variations of the CDW amplitude and the effective mass (assumed to be doping independent here) should be investigated in the future.

Characteristic resistivity associated with a CDW. Despite considerable discussion on the contribution of CDWs to the electronic spectrum [6, 9, 31, 43, 44], the connection between CDWs and charge transport is rarely mentioned because of the difficulty in associating the scattering rate with the CDW. However, our phenomenology provides a simple way to make this connection. Generally, transport dissipation stems from a substantial momentum transfer (e.g., backward scattering), which is a subset of all-microscopic scattering 

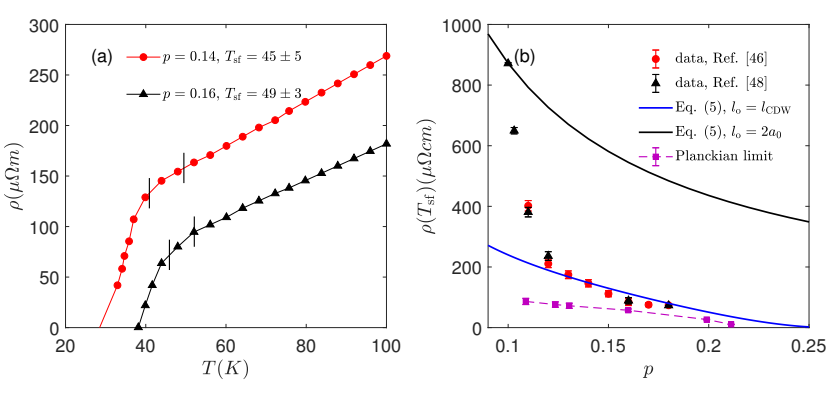

FIG. 4. Characteristic resistivity at the onset temperatures $\left(T_{\mathrm{sf}}\right)$ of the SC fluctuations in Bi-2201. (a) $\rho$ versus $T$. The symbols represent the experimental data obtained in Ref. [46]. The pairs of vertical lines indicate the $T_{\mathrm{sf}}$ error bars determined from Ref. [47]. (b) Doping dependence of $\rho\left(T_{\mathrm{sf}}\right)$. The red and black symbols represent the experimental data reported in Refs. [46, 48]. The solid blue line represents the Eq. (5) prediction with $\gamma_{\Gamma}^{*}=0.11$ and the CDW period $l_{\mathrm{CDW}}$, which is compared to the prediction (solid black line) with the antiferromagnetism (AF) period length $2 a_{0}$, as well as the predictions (purple squares) from Planckian dissipation theory [49] with $m^{*}=2.7 m_{\mathrm{e}}$.

processes [45]. Thus, we assume that the (macroscopic) transport scattering rate is proportional to but smaller than the (microscopic) single-particle scattering rate, $\hbar / 2 \tau=C_{\tau} \Gamma$, where $\tau$ is the relaxation time and $C_{\tau}$ is a dimensionless coefficient less than 1. By substituting $\tau$ and Eq. (1) into the Drude model, we obtain the in-plane resistivity:

$$
\rho_{\mathrm{o}}=\gamma_{\Gamma}^{*} \frac{R_{\mathrm{Q}}}{n_{\mathrm{c}} l_{\mathrm{o}}^{2}}
$$

where $\gamma_{\Gamma}^{*}=4 \pi C_{\tau} \gamma_{\Gamma}, R_{\mathrm{Q}}=h / e^{2}$ is the quantum resistance, $n_{\mathrm{c}}=p K / a_{0} b_{0} c_{0}$ is the carrier density, $a_{0}$ and $b_{0}$ are the in-plane lattice constants, $c_{0}$ is the c-axis lattice constant, $K$ is the number of $\mathrm{Cu}$ or $\mathrm{Fe}$ ions in one unit cell, and $p$ is the carrier concentration per ion.

Eq. (5) quantifies the characteristic resistivity determined by DWO fluctuations. A good candidate for the verification is the underdoped $\mathrm{Bi}-2201$ because its short CDW correlation length fluctuates between 0.75 and 1.5 times the CDW period $l_{\mathrm{CDW}}$ [42], which may efficiently induce scattering. We extract the characteristic resistivity of CDW scattering from previously reported data obtained from high-quality single crystals [46, 48], which have very small residual resistivity at optimum doping. During the extraction, we cautiously avoided the influence of superconductivity (SC) by selecting the "knee" data $\rho\left(T_{\mathrm{sf}}\right)$ at the onset temperature $T_{\mathrm{sf}}$ of the SC fluctuations, as presented in Fig. 4 (a).

In Fig. 4 (b), we fit $\rho\left(T_{\text {sf }}\right)$ with Eq. (5) and the CDW period length $l_{\mathrm{CDW}}=2 \pi / Q_{\mathrm{CDW}}$, where $Q_{\mathrm{CDW}}$ is estimated with the same power law, 0.269[1-(p/0.261 $\left.)^{3.79}\right]$, used in Fig. 3(a). We find that a constant scattering coefficient $\gamma_{\Gamma}^{*}=0.11 \approx J^{2} / t^{2}$ makes the predictions agree
31 well with the data from ten samples between $p=0.12$ and $0.18[46,48]$. This outcome is consistent with the presence of CDW ordering in the $p=0.11-0.16$ region observed by resonant inelastic X-ray scattering [42]. Quantitatively, when the doping increases from 0.12 to $0.18, l_{\mathrm{CDW}}^{2}$ increases by $80 \%$, which is higher than the $50 \%$ increase in the carrier density, revealing that the decrease in $\rho\left(T_{\mathrm{sf}}\right)$ is dominated by the variation in the CDW period length.

It would be helpful to compare the present analysis with other theoretical approaches. In Planckian dissipation theory $[49,50]$, the scattering rate solely determined by temperature is $\hbar / \tau=k_{\mathrm{B}} T$, which predicts the resistivity at $T_{\mathrm{sf}}$ to be $\rho\left(T_{\mathrm{sf}}\right)=\left(m^{*} / n_{\mathrm{c}} e^{2}\right)\left(k_{\mathrm{B}} T_{\mathrm{sf}} / \hbar\right)$. Taking $m^{*}=2.7 m_{\mathrm{e}}$ from optical conductivity measurements [33], this prediction (Fig. 4 (b), purple squares) underestimates the data by nearly $50 \%$. One way to remedy this discrepancy is to attribute it to the "residual resistivity" induced by impurities. However, the stochastic nature of impurities makes the minimum residual resistivity of optimally doped cuprates difficult to explain $[51,52]$. Therefore, the above results reveal that the "knee" resistivity at $T_{\mathrm{sf}}$ in the pseudogap phase has a simple scaling of $\rho\left(T_{\mathrm{sf}}\right) n_{\mathrm{c}} \propto l_{\mathrm{CDW}}^{-2} \propto Q_{\mathrm{CDW}}^{2}$ and thus satisfies the energy law of Eq. (1).

Universal sheet resistance for an antiferromagnetic SDW. An antiferromagnetic (AF) SDW is another widespread DWO in the underdoped regime of HTSCs. Thus, it would be intriguing to examine Eq. (5) to determine whether the AF SDW satisfies the energy law of Eq. (1). In both cuprate- and ironbased HTSCs, the phase transition between AF and $\mathrm{SC}$ is characterized by a low-temperature plateau for the resistivity [48, 53], as shown in Fig. 5 (a). In a superconductor-insulator (SI) transition scenario, the corresponding sheet resistance is predicted to be a universal value, namely, $h / 4 e^{2}=6450 \Omega$ in the Boson localization theory [54]. However, experimental observations revealed that the critical resistance is sample-dependent within a factor of $0.5-2$ of the predicted values, which motivated Goldman's question: "What different physical models govern the various SI transitions which have different critical resistances?" [54]. We now provide a quantitative explanation for this sample-dependent resistance, using Eq. (5).

In our theory, the plateau is attributed to the Umklapp scattering by characteristic fluctuations of the $\mathrm{AF}$ SDW with $l_{\mathrm{o}} \approx 2 a_{0}$. By substituting this $l_{\mathrm{o}}$ into Eq. (5), we predict the critical sheet resistance as follows:

$$
R_{\square}=\frac{\rho}{c_{0} / K}=\frac{\gamma_{\Gamma}^{*}}{p_{\mathrm{c}}} \frac{h}{4 e^{2}},
$$

${ }_{81} \quad$ where $\gamma_{\Gamma}^{*}$ is the corresponding scattering coefficient and ${ }_{82} p_{\mathrm{c}}$ is the critical carrier concentration. If $\gamma_{\Gamma}^{*}$ is univer83 sal, then Eq. (6) predicts that $R_{\square}$ is inversely propor- 

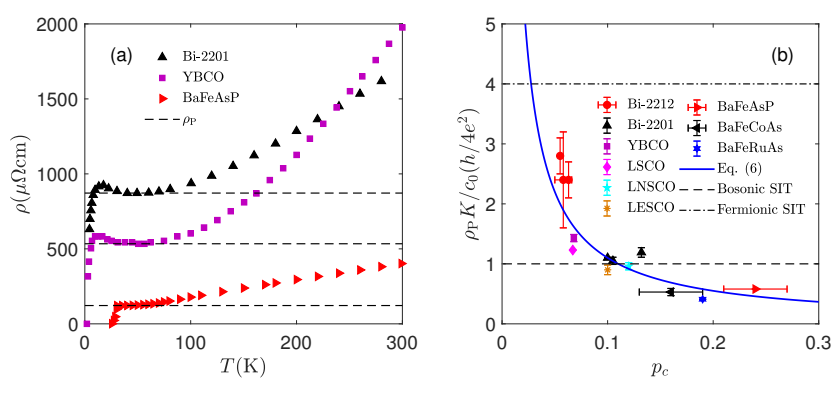

FIG. 5. Critical resistance near the AF-SC transition. (a) $\rho$ versus $T$. The symbols represent experimental data with a low-temperature plateau ( $\rho_{\mathrm{P}}$, dashed lines) near critical doping, as reported in Refs. [46, 48, 53]. (b) Critical sheet resistance of the plateau. The symbols represent the plateau values determined from experimental data reported in Refs. [46, 53, 55-59] (see Supplementary Note 5), and the error bars indicate the plateau determination uncertainty. The solid blue line represents the prediction from Eq. (6) with $\gamma_{\text {SDW }}^{*}=0.11$ and carrier densities and lattice constants consistent with Refs. [58, 60, 61] and Refs. [40, 53, 56, 58, 62]. The dashed and the dash-dotted lines represent the Boson and Fermion localization theories for the SI transition (SIT) [54]. a constant $\gamma_{\Gamma}^{*}=0.11$ is quantitatively consistent with tional to $p_{\mathrm{c}}$. As shown in Fig. 5 (b), the prediction with the reported data for both hole-doped cuprates and iron pnictides within a wide doping range.

The validation of Eq. (6) yields a surprising prediction that the critical sheet resistance per carrier is a universal value, $R_{\square} p_{\mathrm{c}}=\gamma_{\Gamma}^{*} h / 4 e^{2} \approx 710 \Omega$, which represents the true universal feature behind observed critical resistances. This universal sheet resistance of $710 \Omega$ per carrier is not present in the total resistance description $\left(R_{\square}\right)$ by the localization theory [54], and we have achieved a good answer to Goldman's question. Furthermore, since the parents of iron pnictides are metals but not insulators, the validity of Eq. (6) above implies that the energy law is applicable for AF fluctuations in both AF insulator and metal states.

Transition from a CDW to an SDW. It is well known that decreasing doping in cuprates induces an order transition from charge to spin sectors $[1,63]$. Remarkably, the present theory fully confirms this transition from a fluctuating CDW at intermediate doping to an SDW at light doping in the validations of Eqns. (5) to (6) in Fig. 4 and 5. Specifically, Eq. (5) enables us to quantify DWO's period length from resistivity data: $l_{\mathrm{o}}=\sqrt{\gamma_{\Gamma}^{*} R_{\mathrm{Q}} / \rho\left(T_{\mathrm{sf}}\right) n_{\mathrm{c}}}$, where $\rho\left(T_{\mathrm{sf}}\right)$ is observational data. As shown in Fig. 4(b) for Bi-2201, as the doping deceases, $\rho\left(T_{\mathrm{sf}}\right)$ increases sharply from $236 \mu \Omega \mathrm{cm}$ at $p=0.12$ to $871 \mu \Omega \mathrm{cm}$ at $p=0.10$. Taking $\gamma_{\Gamma}^{*}=0.11$, the sharp increases in $\rho\left(T_{\mathrm{sf}}\right)$ is explained by an order's period change from $l_{\mathrm{CDW}} \approx 4 a_{0}$ to $l_{\mathrm{SDW}} \approx 2 a_{0}$, which
32 indicates a possible transition from a CDW-dominated 33 regime to an AF SDW-dominated regime. This asser34 tion is remarkably consistent with an observation from NMR measurements [64] that suggests that the CDW supersedes AF near $p=0.11$ in Bi-2201.

In addition to the consistency for the above-predicted order transition from a CDW to an AF SDW, let us further investigate whether it can also describe the transition in the pseudogap energy scale. Recently, STM measurements of Bi-2201 have exhibited a monotonic increase in the gap energy from $\mathrm{p}=0.12$ to $\mathrm{p}=0.03$, and the absence of a CDW at $\mathrm{p}=0.03$ [24]. The latter suggests that there must be another order dominating the gap in the lightly doped AF regime. According to our theory, SDWs have a larger wavevector (near 0.5) than CDWs (near 0.25), resulting in a larger energy gap, and this finding is qualitatively consistent with the STM observation. Quantitatively, taking $l_{\mathrm{o}}=2 a_{0}$ (the AF period), $\gamma_{\Delta}=0.135$ (the same value as $\mathrm{Pb}-\mathrm{Bi} 2201$ ) and $m^{*}=2.2 \pm 1 m_{\mathrm{e}}$ (from optical conductivity measurements at $\mathrm{p}=0.03[33])$, we predict that $\Delta^{*}=170-470$ $\mathrm{meV}$, which is close to the gap energy scale (i.e., 400-600 $\mathrm{meV}$ ) measured by STM at $p=0.03$. Note that this estimation is much higher than that measured by STM for the CDW $(\lesssim 100 \mathrm{meV})$, which concludes that the pseudogap in the lightly doped regime is indeed determined by the AF SDW.

A further question that our theory should address is whether the CDW extends into the overdoped regime or the energy law is valid across the whole doping range of cuprates [3]. Eq. (3) enables the derivation of DWO length $l_{\mathrm{o}}=h \sqrt{\gamma_{\Delta} / \Delta^{*} m^{*}}$ from the pseudogap energy scale using spectroscopic data. While the pseudogaps in Bi-2201, Bi-2212, and LSCO are experimentally shown to persist up to a high doping level of $p=0.20-0.22[2$, $15,21,65]$, our length mapping formula predicts the existence of CDWs in overdoped cuprates accompanying the pseudogap, consistent with recent STM and RXS measurements of Bi-2201 (up to $p=0.23$ ) and LSCO (up to $p=0.21$ ). On the other hand, Eq. (5) enables us to confirm CDW's dominance from resistivity data for overdoped cuprates, such as $\mathrm{Y}-123[66], \mathrm{Tl}_{2} \mathrm{Ba}_{2} \mathrm{CuO}_{6+\delta}$ [67] and $\mathrm{La}_{2-x} \mathrm{Ce}_{x} \mathrm{CuO}_{4}$ [68], for which the predicted $l_{\mathrm{o}}$ in $p=0.16-0.22$ increases from $3 a_{0}$ to $5.5 a_{0}$. The latter coincides with the characteristic $\mathrm{CDW}$ period $l_{\mathrm{CDW}} \approx$ $4 a_{0}$, indicating the possible presence of a dynamic CDW in the overdoped regime. Note that recent observation of Arpaia et al. indicates that a dynamic CDW pervades the cuprate phase diagrams [69], which is consistent with our assertion.

Then, it is of interest to verify these predictions in other strongly correlated materials using the length mapping formula. A preliminary examination has already yielded consistent outcomes. For instance, from Eq. (3) with $\gamma_{\Delta}=0.135$ and $m^{*}=4 m_{\mathrm{e}}$ (from Ref. [70]), we predict the charge order length for iridate from 
the pseudogap amplitude data (i.e., $70-300 \mathrm{meV}$ ) to be $l_{\mathrm{o}}=1.7-3.6 a_{0}$, consistent with the STM measurement [71].

\section{Discussion}

In summary, complementary to current theories focusing on peculiar intertwined mechanisms of various orders $[8,9,11-13]$, we here uncover a universal energy law linking the pseudogap and the DWO, namely, all three pseudogap energy scales (the scattering rate, the pseudogap energy, and its onset temperature) have a quadratic scaling with the DWO wavevector. All (more than one hundred) single-crystal sample data fully support the present energy law, revealing that the pseudogap originates from the fluctuating DWO, i.e., an SDW at light doping and a CDW at intermediate doping. In our opinion, the universal energy law (and scattering coefficients) represents the zeroth-order relationship between pseudogap energy scales and the DWO wavevector for doping dependence, which provides an important reference point to resolve several conundrums associated with the pseudogap origin.

First, the onset temperature of the pseudogap, if defined as the emergence for the particle-hole pairing of fluctuating SDWs or CDWs, would be naturally higher than the onset temperature of static (coherent) SDWs or CDWs. Second, the universal monotonic decrease in $\Delta^{*}$ and $T^{*}$ with increasing doping stems from the variation in the amplitude and the wavevector of the SDW and CDW. At light doping, the decreases in $\Delta^{*}$ and $T^{*}$ are due to a reduction in the amplitude of the SDW, supported by the observation that the antiferromagnetic spectral weight decreases with doping [23]. On the other hand, at intermediate doping, the decreases are due to reductions in the $Q_{\mathrm{CDW}}$ and the $\mathrm{CDW}$ amplitude, which is supported by spectroscopic measurements [27, 42], as shown in Figs. 2-3 for Bi-2201, Bi-2212 and Hg-1201. In contrast, the La-based cuprates exhibit an increase in $Q_{\mathrm{CDW}}$ with doping (observed in $p=0.115$ $0.21)[27,63,72-74]$ as a consequence of mutual locking of long-range spin and charge orders at low temperatures $[72,75,76]$, a situation that never occurs in other cuprates. The decreases in $\Delta^{*}$ and $T^{*}$ for this exceptional class might be due to the reduction of the CDW amplitude, which is preliminarily observed by the decreasing peak height intensity of X-ray diffraction with doping [74] and will be investigated further in the future. ${ }^{103}$

The present work reports two universal scattering 104 coefficients for the low-energy pseudogap and resistivity, 105 i.e., $\gamma_{\Delta} \approx \gamma_{\Gamma}^{*} \approx J^{2} / t^{2} \approx 0.11$. It is essential to discuss 106 the doping range for the universality of these two ${ }_{107}$ scattering coefficients. Our preliminary understanding 108 is that the universality is preserved for compounds 109 with short-range DWO in the intermediate doping ${ }_{110}$ regime away from both the $\mathrm{AF}$ insulating phase and the ${ }_{111}$ pseudogap QCP. The reason is that the AF insulator ${ }_{112}$ contains long-range AF correlations, leading to stronger scattering behaviour than short-range fluctuations. In contrast, the pseudogap QCP yields a significant decrease in the DWO amplitude, resulting in a weakening of the scattering. As explained in the Methods, we presently use data from high-quality single crystals with the least degree of impurity among all reported data associated with the same compound. However, our theory may be extended to discuss data obtained for less pure samples $[77,78]$ to quantify the additional impurity effect (see Supplemental Note 3). Note that long-range ordering effects in Y-123 and La-based cuprates may significantly affect the magnitudes of the mean-field and fluctuation intensity of DWO (i.e., $\gamma_{\Delta}$ and $\gamma_{\Gamma}^{*}$ ), which is an intriguing issue to be explored in the future.

A further interesting outcome is that the present energy law extends to the strange metal and superconductivity phases. The former comprises fluctuating vortices as an emergent dynamic order, characterized by the thermal de Broglie wavelength $\left(l_{\mathrm{T}} \propto T^{-1 / 2}\right)$ or magnetic length $\left(l_{\mathrm{B}} \propto B^{-1 / 2}\right)$, which scatters carriers with a scattering rate inversely proportional to the square of these lengths [79]. The scattering rate also satisfies the energy law Eq. (1) with a linear dependence on temperature or magnetic field, consistent with recent experimental observations [52, 79-81]. On the other hand, comprehensive measurements have demonstrated a universal linear relation between $T_{\mathrm{c}}$ and the superfluid density $\rho_{\mathrm{s}}$ in most doping regimes of hole-doped cuprates [82-84]. This indicates that the phase coherence energy $k_{\mathrm{B}} T_{\mathrm{C}}$ is inversely proportional to the square of the Cooper-pair distance $\left(l_{\mathrm{p}}=\sqrt{\rho_{\mathrm{s}}}\right)$; thus, Eq. (4) is satisfied. Furthermore, Raman response measurements show that the nodal superconducting gap $\Delta_{\mathrm{SC}}^{\mathrm{N}}$ has the same dome-like doping dependence on $T_{\mathrm{c}}[15]$, indicating $\Delta_{\mathrm{SC}}^{\mathrm{N}} \propto T_{\mathrm{c}} \propto l_{\mathrm{p}}^{-} 2$, again satisfying Eq. (3).

In summary, although originating from different microscopic mechanisms (e.g., the Umklapp scattering by DWO for pseudogap and strong phase fluctuations for superconductivity and strange metal), the characteristic energies (i.e., gap, transition temperature, and scattering rate) of the strange metal, pseudogap, and superconducting phases all satisfy an inverse square scaling on the characteristic lengths of mesoscopic orders (e.g., vortex, DWO, and Cooper pairs). This unified energy law reveals that, regardless of how complex the symmetry-broken forms are, a common invariance constrains the mesoscopic collective electronic motions in cuprates, providing a unified cross-scale link between the microscopic spectrum and macroscopic transport. We speculate that this invariance exhibits an intrinsic quantum nature of strongly correlated electrons and is worthy of further experimental verification and theoretical research in three directions: 
First, it is interesting to verify the energy law for other strongly correlated materials, such as iron-based HTSC, iridate, organic, and heavy Fermion superconductors. Second, we suggest further explorations of the physical origin of the energy law to determine whether there is a local quantum wave state whose single-particle excitation is constrained by the period of a mesoscopic order and thus has quantum kinetic energy determined by the order's period, i.e., $E \propto h^{2} / m^{*} l_{\mathrm{o}}^{2}$. It is highly plausible that the local quantum wave state and the unified energy law can be derived from an action of mesoscopic ordering (e.g., quantum XY model [5]) or the microscopic Hamiltonian (e.g., t-J or Hubbard model) of correlated electrons through some renormalization calculations. Finally, the present universality provides a way to quantify the intertwined behaviours of various forms of collective fluctuations by considering the quantum coupling of multiple scattering channels. This will yield a comprehensive explanation for the preliminary observations that the total scattering rate can be expressed as a coupling formalism of multiple energy laws in recent experiments, e.g., $\sqrt{\left(k_{\mathrm{B}} T\right)^{2}+\left(\mu_{\mathrm{B}} B\right)^{2}}[81,85,86]$, where $\mu_{\mathrm{B}}$ is the Bohr magneton. The progress in these directions will significantly advance the understanding of non-Fermi liquids in strongly correlated electronic systems [87].

\section{Methods}

Characteristic energy scales and the DWO order parameter. In mean-field theory, the characteristic energies that determine the anomalous electronic spectrum and charge transport in the pseudogap phase are described by the single-particle self-energy, which, following Ref. [6], takes the following form:

$$
\Sigma(\mathbf{k}, \omega)=\frac{\Delta_{\mathbf{k}}^{2}}{\omega \pm \epsilon_{\mathbf{k}+\mathbf{Q}}+i \Gamma_{\mathbf{k}}}-i \Gamma_{\mathbf{k}},
$$

Umklapp scattering rate associated with DWO. The energy law for the scattering rate (i.e., Eq. (1)) can be derived from a Umklapp scattering theory for phason modes of DWO [88], assuming a small momentum difference and a large sound velocity. This scattering obeys the conservation of momentum as $\mathbf{k}^{\prime}=\mathbf{k}+\mathbf{q}+n \mathbf{Q}_{\mathrm{o}}$. Following Lee and Rice [88], we obtain the characteristic energy for the mean scattering rate:

$$
\begin{aligned}
& \Gamma=4 \pi m^{*} \sum_{\mathbf{k}, \mathbf{k}}\left(\mathbf{v}_{\mathbf{k}^{\prime}}-\mathbf{v}_{\mathbf{k}}\right)^{2} W_{\mathbf{k}, \mathbf{k}^{\prime}}\left[\delta\left(E_{\mathbf{k}}-E_{\mathbf{k}^{\prime}}-\omega_{\mathbf{q}}\right) \frac{\partial f}{\partial E}\right. \\
& \left.\left(1+n_{\mathbf{q}}-f_{\mathbf{k}^{\prime}}\right)+\delta\left(E_{\mathbf{k}}-E_{\mathbf{k}^{\prime}}+\omega_{\mathbf{q}}\right) \frac{\partial f}{\partial E^{\prime}}\left(1+n_{\mathbf{q}}-f_{\mathbf{k}}\right)\right],
\end{aligned}
$$

where $\mathbf{v}$ and $E$ are the velocity and energy of a carrier, respectively; $W_{\mathbf{k}, \mathbf{k}^{\prime}}$ is the module square of the transition matrix element describing the carrier-order coupling, $\omega_{\mathbf{q}}$ is the frequency of the phason; and $f$ and $n_{\mathbf{q}}$ are the distribution functions of the carrier and phason modes, respectively.

For a small momentum difference, it is reasonable to assume a linear relation, i.e., $\left(\mathbf{v}_{\mathbf{k}^{\prime}}-\mathbf{v}_{\mathbf{k}}\right) \approx s \hbar\left(\mathbf{k}-\mathbf{k}^{\prime}\right) / m^{*}$, where $s$ is a dimensionless constant. Furthermore, we assume that, compared to $Q_{\mathrm{o}}$, the $q$ of low-lying excitations is small, yielding $\left(\mathbf{v}_{\mathbf{k}^{\prime}}-\mathbf{v}_{\mathbf{k}}\right)^{2} \approx n^{2} s^{2} \hbar^{2} Q_{\mathrm{o}}^{2} /\left(m^{*}\right)^{2}$. By substituting this expression into Eq. (9), we obtain Eq. (1) and a dimensionless coefficient describing the mean strength of carrier-phason scattering:

$$
\begin{aligned}
\gamma_{\Gamma}= & \frac{4}{\pi} n^{2} s^{2} \sum_{\mathbf{k}^{\prime}, \mathbf{k}} W_{\mathbf{k}, \mathbf{k}^{\prime}}\left[\delta\left(E_{\mathbf{k}}-E_{\mathbf{k}^{\prime}}-\omega_{\mathbf{q}}\right) \frac{\partial f}{\partial E}\left(1+n_{\mathbf{q}}-f_{\mathbf{k}^{\prime}}\right)\right. \\
& \left.+\delta\left(E_{\mathbf{k}}-E_{\mathbf{k}^{\prime}}+\omega_{\mathbf{q}}\right) \frac{\partial f}{\partial E^{\prime}}\left(1+n_{\mathbf{q}}-f_{\mathbf{k}}\right)\right]
\end{aligned}
$$

Model the doping dependence of $Q_{\mathrm{CDW}}$. To make continuous predictions dependence of the gap energy, the scattering rate, and the resistivity on doping, we propose a least-squares fit for the doping-dependent phase-averaged $Q_{\mathrm{CDW}}$ data. During the fitting procedure, we assume that the amplitude of the fluctuating $\mathrm{CDW}$ is nonzero in the doping range considered in this work. Under the constraints of simplicity and physical consistency, we found that the linear fit and the defect power law are the two most suitable fitting functions for Hg-2201, Bi-2212, and Bi-2201, as shown in Fig. S1 (see Supplementary Note 1). For instance, although a polynomial provides a more accurate fit for $Q_{\mathrm{CDW}}$ of $\mathrm{Bi}-2201$, it is inconsistent with the observation that the CDW is nearly commensurate in the lightly doped Bi-2201 [21, 24]. In contrast, a defect power law, i.e., $Q_{\mathrm{CDW}}=0.269\left[1-(p / 0.261)^{3.79}\right]$, is the simplest function consistent with this scenario.

The choice of validating samples. To date, there have been thousands of reported experimental measurements devoted to the pseudogap, DWO, and 
resistivity, involving considerable diversity, compound series, doping regimes, and sample qualities. To identify a universal energy law, we restrict ourselves mainly to high-quality single crystals with short-range DWO to remove various higher-order effects, e.g., excessive impurities and the long-range ordering effect. Therefore, in this paper, the samples selected for validation are high-quality single-crystal series of hole-doped Bi-2201, Bi-2212, and Hg-1201 prepared by highly respected experimental groups for their strong two-dimensional nature and systematic observations of short-range DWO $([14,15,27,37,42])$, as well as lowest reported resistivity values among all reported data associated with the same compound (e.g., Bi-2201 prepared by Ando's group [46, 51]). Therefore, the choice of our validating samples is not arbitrary but consistent with our strict theoretical requirements. Please refer to Supplementary Notes 4 and 5 for the corresponding list of the data sources used in this work.

\section{Acknowledgements}

We thank J. E. Hoffman and T. A. Webb for their helpful discussions. This work was partially supported by the National Natural Science Foundation of China (Grant No. 11452002).

\section{Author contributions}

Both authors conceived the original idea and wrote the paper. Z.S. supervised the project. R.L. performed the data analysis.

* Corresponding author; she@pku.edu.cn

[1] B. Keimer, S. A. Kivelson, M. R. Norman, S. Uchida, and J. Zaanen, From quantum matter to hightemperature superconductivity in copper oxides, Nature 518, 179 (2015).

[2] A. Kordyuk, Pseudogap from arpes experiment: three gaps in cuprates and topological superconductivity, Low Temp. Phys. 41, 319 (2015).

[3] A. Frano, S. Blanco-Canosa, B. Keimer, and R. J. Birgeneau, Charge ordering in superconducting copper oxides, J. Phys. Condens. Matter 32, 374005 (2020).

[4] Y. Sato, S. Kasahara, H. Murayama, Y. Kasahara, E. G. Moon, T. Nishizaki, T. Loew, J. Porras, B. Keimer, ${ }_{107}$ T. Shibauchi, and Y. Matsuda, Thermodynamic evi- 108 dence for a nematic phase transition at the onset of the 109 pseudogap in $\mathrm{YBa}_{2} \mathrm{Cu}_{3} \mathrm{O}_{y}$, Nat. Phys. 13, 1074 (2017). ${ }_{110}$

[5] C. M. Varma, Colloquium: Linear in temperature resis- 111 tivity and associated mysteries including high temper- 112 ature superconductivity, Rev. Mod. Phys. 92, 031001113 (2020).

[6] M. R. Norman, A. Kanigel, M. Randeria, U. Chatter- 115 jee, and J. C. Campuzano, Modeling the Fermi arc in ${ }_{116}$ underdoped cuprates, Phys. Rev. B 76, 174501 (2007). ${ }_{117}$

[7] S. E. Sebastian and C. Proust, Quantum oscillations in 118 hole-doped cuprates, Annu. Rev. Condens. Matter Phys. ${ }_{119}$
6, $411(2015)$

[8] D. F. Agterberg, J. C. S. Davis, S. D. Edkins, E. Fradkin, D. J. Van Harlingen, S. A. Kivelson, P. A. Lee, L. Radzihovsky, J. M. Tranquada, and Y. Wang, The physics of pair-density waves: Cuprate superconductors and beyond, Annu. Rev. Condens. Matter Phys. 11, 231 (2020).

[9] C. M. Varma, Pseudogap and Fermi arcs in underdoped cuprates, Phys. Rev. B 99, 224516 (2019).

[10] S.-i. Uchida, Identifying the pseudogap in cuprates with a nematic phase, J. Phys. Soc. Jpn. 17, 07 (2020).

[11] E. Fradkin, S. A. Kivelson, and J. M. Tranquada, Colloquium: Theory of intertwined orders in high temperature superconductors, Rev. Mod. Phys. 87, 457 (2015).

[12] R. M. Fernandes, P. P. Orth, and J. Schmalian, Intertwined vestigial order in quantum materials: Nematicity and beyond, Annu. Rev. Condens. Matter Phys. 10, 133 (2019).

[13] L. Nie, A. V. Maharaj, E. Fradkin, and S. A. Kivelson, Vestigial nematicity from spin and/or charge order in the cuprates, Phys. Rev. B 96, 085142 (2017).

[14] B. Loret, N. Auvray, Y. Gallais, M. Cazayous, A. Forget, D. Colson, M. H. Julien, I. Paul, M. Civelli, and A. Sacuto, Intimate link between charge density wave, pseudogap and superconducting energy scales in cuprates, Nat. Phys. 15, 771 (2019).

[15] B. Loret, N. Auvray, G. D. Gu, A. Forget, D. Colson, M. Cazayous, Y. Gallais, I. Paul, M. Civelli, and A. Sacuto, Universal relationship between the energy scales of the pseudogap phase, the superconducting state, and the charge-density-wave order in copper oxide superconductors, Phys. Rev. B 101, 214520 (2020).

[16] S. Mukhopadhyay, R. Sharma, C. K. Kim, S. D. Edkins, M. H. Hamidian, H. Eisaki, S.-i. Uchida, E.-A. Kim, M. J. Lawler, A. P. Mackenzie, J. C. S. Davis, and K. Fujita, Evidence for a vestigial nematic state in the cuprate pseudogap phase, Proc. Nat. Acad. Sci. USA 116, 13249 (2019).

[17] A. Mesaros, K. Fujita, S. D. Edkins, M. H. Hamidian, H. Eisaki, S. I. Uchida, J. C. S. Davis, M. J. Lawler, and E. A. Kim, Commensurate $4 a_{0}$-period charge density modulations throughout the $\mathrm{Bi}_{2} \mathrm{Sr}_{2} \mathrm{CaCu}_{2} \mathrm{O}_{8+x}$ pseudogap regime, Proc. Natl. Acad. Sci. USA 113, 12661 (2016).

[18] H. Zhao, Z. Ren, B. Rachmilowitz, J. Schneeloch, R. Zhong, G. Gu, Z. Wang, and I. Zeljkovic, Chargestripe crystal phase in an insulating cuprate, Nat. Mater. 18, 103 (2019).

[19] Y. Zhang, A. Mesaros, K. Fujita, S. D. Edkins, M. H. Hamidian, K. Ch'ng, H. Eisaki, S. Uchida, J. C. S. Davis, E. Khatami, and E.-A. Kim, Machine learning in electronic-quantum-matter imaging experiments, Nature 570, 484 (2019).

[20] I. Vinograd, R. Zhou, M. Hirata, T. Wu, H. Mayaffre, S. Krämer, R. Liang, W. N. Hardy, D. A. Bonn, and M.-H. Julien, Locally commensurate charge-density wave with three-unit-cell periodicity in $\mathrm{YBa}_{2} \mathrm{Cu}_{3} \mathrm{O}_{y}$, Nat. Commun. 12, 3274 (2021).

[21] T. A. Webb, M. C. Boyer, Y. Yin, D. Chowdhury, Y. He, T. Kondo, T. Takeuchi, H. Ikuta, E. W. Hudson, J. E. Hoffman, and M. H. Hamidian, Density Wave Probes Cuprate Quantum Phase Transition, Phys. Rev. X 9, 021021 (2019).

[22] S. A. Kivelson and S. Lederer, Linking the pseudogap 
in the cuprates with local symmetry breaking: A commentary, Proc. Nat. Acad. Sci. USA 116, 14395 (2019).

23] M. Fujita, H. Hiraka, M. Matsuda, M. Matsuura, J. M. Tranquada, S. Wakimoto, G. Xu, and K. Yamada, Progress in Neutron Scattering Studies of Spin Excitations in High- $T_{c}$ Cuprates, J. Phys. Soc. Jpn. 81, 011007 (2012).

[24] P. Cai, W. Ruan, Y. Y. Peng, C. Ye, X. T. Li, Z. Q. Hao, X. J. Zhou, D. H. Lee, and Y. Y. Wang, Visualizing the evolution from the Mott insulator to a charge-ordered insulator in lightly doped cuprates, Nat. Phys. 12, 1047 (2016).

[25] Z.-S. She, X. Chen, and F. Hussain, Quantifying wall turbulence via a symmetry approach: a lie group theory, J. Fluid Mech. 827, 322 (2017).

[26] X. Chen, F. Hussain, and Z.-S. She, Quantifying wall turbulence via a symmetry approach. Part 2 . Reynolds stresses, J. Fluid Mech. 850, 401 (2018).

[27] R. Comin and A. Damascelli, Resonant X-Ray Scattering Studies of Charge Order in Cuprates, Annu. Rev. Condens. Matter Phys. 7, 369 (2016).

[28] P. A. Lee, N. Nagaosa, and X. G. Wen, Doping a Mott insulator: Physics of high-temperature superconductivity, Rev. Mod. Phys. 78, 17 (2006).

[29] M. S. Hybertsen, E. B. Stechel, M. Schluter, and D. R. Jennison, Renormalization from density-functional theory to strong-coupling models for electronic states in Cu-O materials, Phys. Rev. B 41, 11068 (1990).

[30] P. E. Sulewski, P. A. Fleury, K. B. Lyons, S. W. Cheong, and Z. Fisk, Light scattering from quantum spin fluctuations in $\mathrm{R}_{2} \mathrm{CuO}_{4}(\mathrm{R}=\mathrm{La}, \mathrm{Nd}, \mathrm{Sm})$, Phys. Rev. B 41, 225 (1990).

[31] Y. Wang and A. Chubukov, Charge-density-wave order with momentum $(2 \mathrm{Q}, 0)$ and $(0,2 \mathrm{Q})$ within the spin-fermion model: Continuous and discrete symmetry breaking, preemptive composite order, and relation to pseudogap in hole-doped cuprates, Phys. Rev. B 90, 035149 (2014).

[32] T. Yoshida, W. Malaeb, S. Ideta, D. H. Lu, R. G. Moor, Z. X. Shen, M. Okawa, T. Kiss, K. Ishizaka, S. Shin, S. Komiya, Y. Ando, H. Eisaki, S. Uchida, and A. Fujimori, Coexistence of a pseudogap and a superconducting gap for the high- $T_{c}$ superconductor $\mathrm{La}_{2-x} \mathrm{Sr}_{x} \mathrm{CuO}_{4}$ studied by angle-resolved photoemission spectroscopy, Phys. Rev. B 93, 014513 (2016).

[33] Y. M. Dai, B. Xu, P. Cheng, H. Q. Luo, H. H. Wen, 110 X. G. Qiu, and R. P. S. M. Lobo, Doping evolution 111 of the optical scattering rate and effective mass of 112 $\mathrm{Bi}_{2} \mathrm{Sr}_{2-x} \mathrm{La}_{x} \mathrm{CuO}_{6}$, Phys. Rev. B 85, 092504 (2012). ${ }_{113}$

[34] R. Comin, A. Frano, M. M. Yee, Y. Yoshida, H. Eisaki, 114 E. Schierle, E. Weschke, R. Sutarto, F. He, A. Soumya- 115 narayanan, Y. He, M. Le Tacon, I. S. Elfimov, J. E. 116 Hoffman, G. A. Sawatzky, B. Keimer, and A. Damas- 117 celli, Charge Order Driven by Fermi-Arc Instability in 118 $\mathrm{Bi}_{2} \mathrm{Sr}_{2-z} \mathrm{La}_{z} \mathrm{CuO}_{6+\delta}$, Science 343, 390 (2014).

[35] W. D. Wise, K. Chatterjee, M. C. Boyer, T. Kondo, 120 T. Takeuchi, H. Ikuta, Z. Xu, J. Wen, G. D. Gu, 121 Y. Wang, and E. W. Hudson, Imaging nanoscale Fermi- ${ }_{122}$ surface variations in an inhomogeneous superconductor, 123 Nat. Phys. 5, 213 (2009).

[36] N. Barišić, S. Badoux, M. K. Chan, C. Dorow, W. Tabis, ${ }_{125}$ B. Vignolle, G. Yu, J. Béard, X. Zhao, C. Proust, and 126 M. Greven, Universal quantum oscillations in the un- 127 derdoped cuprate superconductors, Nat. Phys. 9, $761{ }_{128}$
(2013).

[37] W. Tabis, B. Yu, I. Bialo, M. Bluschke, T. Kolodziej, A. Kozlowski, E. Blackburn, K. Sen, E. M. Forgan, M. Von Zimmermann, Y. Tang, E. Weschke, B. Vignolle, M. Hepting, H. Gretarsson, R. Sutarto, F. He, M. Le Tacon, N. Barisic, G. Yu, and M. Greven, Synchrotron x-ray scattering study of charge-density-wave order in $\mathrm{HgBa}_{2} \mathrm{CuO}_{4+\delta}$, Phys. Rev. B 96, 12 (2017).

[38] G. Campi, A. Bianconi, N. Poccia, G. Bianconi, L. Barba, G. Arrighetti, D. Innocenti, J. Karpinski, N. D. Zhigadlo, S. M. Kazakov, M. Burghammer, M. V. Zimmermann, M. Sprung, and A. Ricci, Inhomogeneity of charge-density-wave order and quenched disorder in a high- $T_{c}$ superconductor, Nature 525, 359 (2015).

[39] J. C. Campuzano, H. Ding, M. R. Norman, H. M. Fretwell, M. Randeria, A. Kaminski, J. Mesot, T. Takeuchi, T. Sato, T. Yokoya, T. Takahashi, T. Mochiku, K. Kadowaki, P. Guptasarma, D. G. Hinks, Z. Konstantinovic, Z. Z. Li, and H. Raffy, Electronic spectra and their relation to the $(\pi, \pi)$ collective mode in high- $T_{c}$ superconductors, Phys. Rev. Lett. 83, 3709 (1999).

[40] W. Zhou and W. Liang, Fundamental Research of HighTemperature Superconductor (Shanghai Science Press, Shanghai, 1999).

[41] Y. He, Y. Yin, M. Zech, A. Soumyanarayanan, M. M. Yee, T. Williams, M. C. Boyer, K. Chatterjee, W. D. Wise, I. Zeljkovic, T. Kondo, T. Takeuchi, H. Ikuta, P. Mistark, R. S. Markiewicz, A. Bansil, S. Sachdev, E. W. Hudson, and J. E. Hoffman, Fermi surface and pseudogap evolution in a cuprate superconductor, Science 344, 608 (2014).

[42] Y. Y. Peng, R. Fumagalli, Y. Ding, M. Minola, S. Caprara, D. Betto, M. Bluschke, G. M. De Luca, K. Kummer, E. Lefrancois, M. Salluzzo, H. Suzuki, M. Le Tacon, X. J. Zhou, N. B. Brookes, B. Keimer, L. Braicovich, M. Grilli, and G. Ghiringhelli, Re-entrant charge order in overdoped $(\mathrm{Bi}, \mathrm{Pb})_{2.12} \mathrm{Sr}_{1.88} \mathrm{CuO}_{6+\delta}$ outside the pseudogap regime, Nat. Mater. 17, 697 (2018).

[43] S. Chakravarty, R. B. Laughlin, D. K. Morr, and C. Nayak, Hidden order in the cuprates, Phys. Rev. B 63, 094503 (2001).

[44] K.-Y. Yang, T. M. Rice, and F.-C. Zhang, Phenomenological theory of the pseudogap state, Phys. Rev. B 73, 174501 (2006).

[45] E. Abrahams and C. M. Varma, What angle-resolved photoemission experiments tell about the microscopic theory for high-temperature superconductors, Proc. Nat. Acad. Sci. USA 97, 5714 (2000).

[46] Y. Ando, S. Komiya, K. Segawa, S. Ono, and Y. Kurita, Electronic Phase Diagram of High- $T_{c}$ Cuprate Superconductors from a Mapping of the In-Plane Resistivity Curvature, Phy. Rev. Lett. 93, 267001 (2004).

[47] H. H. Wen, G. Mu, H. Luo, H. Yang, L. Shan, C. Ren, P. Cheng, J. Yan, and L. Fang, Specific-heat measurement of a residual superconducting state in the normal state of underdoped $\mathrm{Bi}_{2} \mathrm{Sr}_{2-x} \mathrm{La}_{x} \mathrm{CuO}_{6+\delta}$ cuprate superconductors, Phys. Rev. Lett. 103, 067002 (2009).

[48] S. Ono, Y. Ando, T. Murayama, F. F. Balakirev, J. B. Betts, and G. S. Boebinger, Metal-to-insulator crossover in the low-temperature normal state of $\mathrm{Bi}_{2} \mathrm{Sr}_{2-x} \mathrm{La}_{x} \mathrm{CuO}_{6+\delta}$, Phys. Rev. Lett. 85, 638 (2000).

[49] A. Legros, S. Benhabib, W. Tabis, F. Laliberté, M. Dion, M. Lizaire, B. Vignolle, D. Vignolles, H. Raffy, Z. Z. 
Li, P. Auban-Senzier, N. Doiron-Leyraud, P. Fournier, D. Colson, L. Taillefer, and C. Proust, Universal Tlinear resistivity and Planckian dissipation in overdoped cuprates, Nat. Phys. 15, 142 (2019).

[50] J. Zaanen, Why the temperature is high, Nature 430, $512(2004)$.

[51] Y. Ando, Y. Hanaki, S. Ono, T. Murayama, K. Segawa, N. Miyamoto, and S. Komiya, Carrier concentrations in $\mathrm{Bi}_{2} \mathrm{Sr}_{2-z} \mathrm{La}_{z} \mathrm{CuO}_{6+\delta}$ single crystals and their relation to the Hall coefficient and thermopower, Phys. Rev. B 61, R14956 (2000).

[52] P. Giraldo-Gallo, J. Galvis, Z. Stegen, K. Modic, F. Balakirev, J. Betts, X. Lian, C. Moir, S. Riggs, and J. Wu, Scale-invariant magnetoresistance in a cuprate superconductor, Science 361, 479 (2018).

[53] S. Kasahara, T. Shibauchi, K. Hashimoto, K. Ikada, S. Tonegawa, R. Okazaki, H. Shishido, H. Ikeda, H. Takeya, K. Hirata, T. Terashima, and Y. Matsuda, Evolution from non-Fermi- to Fermi-liquid transport via isovalent doping in $\mathrm{BaFe}_{2}\left(\mathrm{As}_{1-x} \mathrm{P}_{x}\right)_{2}$ superconductors, Phys. Rev. B 81, 184519 (2010).

[54] A. M. Goldman, Superconductor-insulator transitions, Int. J. Mod. Phys. B 24, 4081 (2010).

[55] S. Komiya, H.-D. Chen, S.-C. Zhang, and Y. Ando, Magic Doping Fractions for High-Temperature Superconductors, Phys. Rev. Lett. 94, 207004 (2005).

[56] D. Mandrus, L. Forro, C. Kendziora, and L. Mihaly, Two-dimensional electron localization in bulk single crystals of $\mathrm{Bi}_{2} \mathrm{Sr}_{2} \mathrm{Y}_{x} \mathrm{Ca}_{1-x} \mathrm{Cu}_{2} \mathrm{O}_{8}$, Phys. Rev. B 44, 2418 (1991).

[57] P. Orgiani, C. Aruta, G. Balestrino, D. Born, L. Maritato, P. G. Medaglia, D. Stornaiuolo, F. Tafuri, and A. Tebano, Direct measurement of sheet resistance Rsquare in cuprate systems: evidence of a fermionic scenario in a metal-insulator transition, Phys. Rev. Lett. 98, 036401 (2007).

[58] F. Rullier-Albenque, D. Colson, A. Forget, P. Thuéry, 101 and S. Poissonnet, Hole and electron contributions to 102 the transport properties of $\mathrm{Ba}\left(\mathrm{Fe}_{1-x} \mathrm{Ru}_{x}\right)_{2} \mathrm{As}_{2}$ single ${ }_{103}$ crystals, Phys. Rev. B 81, 224503 (2010).

[59] Z. Shi, P. G. Baity, T. Sasagawa, and D. Popovic, Vortex 105 phase diagram and the normal state of cuprates with 106 charge and spin orders, Sci. Adv. 6, aay8946 (2020). 107

[60] F. Rullier-Albenque, Influence of the electronic structure 108 on the transport properties of some iron pnictides, C. R. ${ }_{109}$ Physique 17, 164 (2016).

[61] Z. R. Ye, Y. Zhang, F. Chen, M. Xu, Q. Q. Ge, J. Jiang, 111 B. P. Xie, and D. L. Feng, Doping dependence of ${ }_{112}$ the electronic structure in phosphorus-doped ferropnic- 113 tide superconductor $\mathrm{BaFe}_{2}\left(\mathrm{As}_{1-x} \mathrm{P}_{x}\right)_{2}$ studied by angle- ${ }_{114}$ resolved photoemission spectroscopy, Phys. Rev. B 86, 115 035136 (2012).

[62] A. Sawa, M. Kawasaki, H. Takagi, and Y. Tokura, ${ }_{117}$ Electron-doped superconductor $\mathrm{La}_{2 x} \mathrm{Ce}_{x} \mathrm{CuO}_{4}$ : Prepa- ${ }_{118}$ ration of thin films and modified doping range for su- 119 perconductivity, Phys. Rev. B 66, 014531 (2002). 120

[63] J. J. Wen, H. Huang, S. J. Lee, H. Jang, J. Knight, 121 Y. S. Lee, M. Fujita, K. M. Suzuki, S. Asano, S. A. ${ }_{122}$ Kivelson, C. C. Kao, and J. S. Lee, Observation of two 123 types of charge-density-wave orders in superconducting 124 $\mathrm{La}_{2-x} \mathrm{Sr}_{x} \mathrm{CuO}_{4}$, Nat. Commun. 10, 3269 (2019).

[64] S. Kawasaki, Z. Li, M. Kitahashi, C. T. Lin, P. L. Kuhns, ${ }_{126}$ A. P. Reyes, and G. Q. Zheng, Charge-density-wave or- 127 der takes over antiferromagnetism in $\mathrm{Bi}_{2} \mathrm{Sr}_{2-x} \mathrm{La}_{x} \mathrm{CuO}_{6} \quad 128$ superconductors, Nat. Commun. 8, 1267 (2017).

[65] M. Hashimoto, I. M. Vishik, R.-H. He, T. P. Devereaux, and Z.-X. Shen, Energy gaps in high-transitiontemperature cuprate superconductors, Nat. Phys. 10, 483 (2014).

[66] M. S. Grbić, M. Požek, D. Paar, V. Hinkov, M. Raichle, D. Haug, B. Keimer, N. Barišić, and A. Dulčić, Temperature range of superconducting fluctuations above $T_{c}$ in $\mathrm{YBa}_{2} \mathrm{Cu}_{3} \mathrm{O}_{7-\delta}$ single crystals, Phys. Rev. B 83, 144508 (2011).

[67] M. S. Grbić, N. Barišić, A. Dulčić, I. Kupčić, Y. Li, X. Zhao, G. Yu, M. Dressel, M. Greven, and M. Požek, Microwave measurements of the in-plane and c-axis conductivity in $\mathrm{HgBa}_{2} \mathrm{CuO}_{4+\delta}$ : Discriminating between superconducting fluctuations and pseudogap effects, Phys. Rev. B 80, 094511 (2009).

[68] T. Sarkar, P. R. Mandal, J. S. Higgins, Y. Zhao, H. Yu, K. Jin, and R. L. Greene, Fermi surface reconstruction and anomalous low-temperature resistivity in electrondoped $\mathrm{La}_{2 x} \mathrm{Ce}_{x} \mathrm{CuO}_{4}$, Phys. Rev. B 96, 155449 (2017).

[69] R. Arpaia, S. Caprara, R. Fumagalli, G. De Vecchi, Y. Y. Peng, E. Andersson, D. Betto, G. M. De Luca, N. B. Brookes, F. Lombardi, M. Salluzzo, L. Braicovich, C. Di Castro, M. Grilli, and G. Ghiringhelli, Dynamical charge density fluctuations pervading the phase diagram of a Cu-based high- $T_{c}$ superconductor, Science 365, 906 (2019).

[70] S. J. Moon, H. Jin, K. W. Kim, W. S. Choi, Y. S. Lee, J. Yu, G. Cao, A. Sumi, H. Funakubo, C. Bernhard, and T. W. Noh, Dimensionality-controlled insulator-metal transition and correlated metallic state in $5 \mathrm{~d}$ transition metal oxides $\mathrm{Sr}_{n+1} \operatorname{Ir}_{n} \mathrm{O}_{3 n+1}(\mathrm{n}=1,2$, and infinity), Phys. Rev. Lett. 101, 226402 (2008).

[71] I. Battisti, K. M. Bastiaans, V. Fedoseev, A. de la Torre, N. Iliopoulos, A. Tamai, E. C. Hunter, R. S. Perry, J. Zaanen, F. Baumberger, and M. P. Allan, Universality of pseudogap and emergent order in lightly doped mott insulators, Nat. Phys. 13, 21 (2017).

[72] H. Miao, R. Fumagalli, M. Rossi, J. Lorenzana, G. Seibold, F. Yakhou-Harris, K. Kummer, N. Brookes, G. Gu, L. Braicovich, G. Ghiringhelli, and M. Dean, Formation of incommensurate charge density waves in cuprates, Phys. Rev. X 9, 031042 (2019).

[73] J. Lin, H. Miao, D. Mazzone, G. Gu, A. Nag, A. Walters, M. García-Fernández, A. Barbour, J. Pelliciari, I. Jarrige, M. Oda, K. Kurosawa, N. Momono, K.-J. Zhou, V. Bisogni, X. Liu, and M. Dean, Strongly Correlated Charge Density Wave in $\mathrm{La}_{2-x} \mathrm{Sr}_{x} \mathrm{CuO}_{4}$ Evidenced by Doping-Dependent Phonon Anomaly, Phys. Rev. Lett. 124, 207005 (2020).

[74] H. Miao, G. Fabbris, R. J. Koch, D. G. Mazzone, C. S. Nelson, R. Acevedo-Esteves, G. D. Gu, Y. Li, T. Yilimaz, K. Kaznatcheev, E. Vescovo, M. Oda, T. Kurosawa, N. Momono, T. Assefa, I. K. Robinson, E. S. Bozin, J. M. Tranquada, P. D. Johnson, and M. P. M. Dean, Charge density waves in cuprate superconductors beyond the critical doping, npj Quantum Mater. 6, 31 (2021).

[75] H. Miao, J. Lorenzana, G. Seibold, Y. Y. Peng, A. Amorese, F. Yakhou-Harris, K. Kummer, N. B. Brookes, R. M. Konik, V. Thampy, G. D. Gu, G. Ghiringhelli, L. Braicovich, and M. P. M. Dean, High-temperature charge density wave correlations in $\mathrm{La}_{1.875} \mathrm{Ba}_{0.125} \mathrm{CuO}_{4}$ without spin-charge locking, Proc. 
Nat. Acad. Sci. USA 114, 12430 (2017).

[76] H. Miao, D. Ishikawa, R. Heid, M. Le Tacon, G. Fabbris, D. Meyers, G. Gu, A. Baron, and M. Dean, Incommensurate phonon anomaly and the nature of charge density waves in cuprates, Phys. Rev. X 8, 011008 (2018).

[77] Y. Ando, G. S. Boebinger, A. Passner, N. L. Wang, C. Geibel, and F. Steglich, Metallic in-plane and divergent out-of-plane resistivity of a high- $T_{c}$ cuprate in the zero-temperature limit, Phy. Rev. Lett. 77, 2065 (1996).

[78] J. Meng, G. Liu, W. Zhang, L. Zhao, H. Liu, W. Lu, X. Dong, and X. J. Zhou, Growth, characterization and physical properties of high-quality large single crystals of $\mathrm{Bi}_{2} \mathrm{Sr}_{2-x} \mathrm{La}_{x} \mathrm{CuO}_{6}$ high-temperature superconductors, Supercond. Sci. Technol. 22, 045010 (2009).

[79] R. Li and Z.-S. She, Emergent mesoscopic quantum vortex and planckian dissipation in the strange metal phase, New J. Phys. 23, 043050 (2021).

[80] J. A. N. Bruin, H. Sakai, R. S. Perry, and A. P. Mackenzie, Similarity of scattering rates in metals showing tlinear resistivity, Science 339, 804 (2013).

[81] I. M. Hayes, R. D. McDonald, N. P. Breznay, T. Helm, 5 P. J. W. Moll, M. Wartenbe, A. Shekhter, and J. G. Analytis, Scaling between magnetic field and temperature in the high-temperature superconductor $\mathrm{BaFe}_{2}\left(\mathrm{As}_{1-x} \mathrm{P}_{x}\right)_{2}$, Nat. Phys. 12, 916 (2016).

[82] Y. J. Uemura, G. M. Luke, B. J. Sternlieb, J. H. Brewer, J. F. Carolan, W. N. Hardy, R. Kadono, J. R. Kempton, R. F. Kiefl, S. R. Kreitzman, P. Mulhern, T. M. Riseman, D. L. Williams, B. X. Yang, S. Uchida, H. Takagi,
J. Gopalakrishnan, A. W. Sleight, M. A. Subramanian, C. L. Chien, M. Z. Cieplak, G. Xiao, V. Y. Lee, B. W. Statt, C. E. Stronach, W. J. Kossler, and X. H. Yu, Universal Correlations between $T_{c}$ and $n_{s} / m^{*}$ (Carrier Density over Effective Mass) in High- $T_{c}$ Cuprate Superconductors, Phys. Rev. Lett. 62, 2317 (1989).

[83] I. Božović, X. He, J. Wu, and A. T. Bollinger, Dependence of the critical temperature in overdoped copper oxides on superfluid density, Nature 536, 309 (2016).

[84] I. Božović, X. He, J. Wu, and A. T. Bollinger, The vanishing superfluid density in cuprates - and why it matters, Journal of Super. And Novel Mag. 31, 2683 (2018).

[85] S. Licciardello, N. Maksimovic, J. Ayres, J. Buhot, M. Čulo, B. Bryant, S. Kasahara, Y. Matsuda, T. Shibauchi, V. Nagarajan, J. G. Analytis, and N. E. Hussey, Coexistence of orbital and quantum critical magnetoresistance in $\mathrm{FeSe}_{1-x} \mathrm{~S}_{x}$, Phys. Rev. Research. 1, 023011 (2019).

[86] Y. Nakajima, T. Metz, C. Eckberg, K. Kirshenbaum, A. Hughes, R. Wang, L. Wang, S. R. Saha, I. L. Liu, N. P. Butch, D. Campbell, Y. S. Eo, D. Graf, Z. Liu, S. V. Borisenko, P. Y. Zavalij, and J. Paglione, Quantum-critical scale invariance in a transition metal alloy, Commun. Phys. 3, 181 (2020).

[87] S.-S. Lee, Recent developments in non-fermi liquid theory, Annu. Rev. Condens. Matter Phys. 9, 227 (2018).

[88] P. A. Lee and T. M. Rice, Electric field depinning of charge density waves, Phys. Rev. B 19, 3970 (1979). 


\section{Supplementary Files}

This is a list of supplementary files associated with this preprint. Click to download.

- SM.pdf 